\title{
An Efficient Algorithm for Generalized Polynomial Partitioning and Its Applications
}

\author{
Pankaj K. Agarwal
}

Department of Computer Science, Duke University, Box 90129, Durham, NC 27708-0129 USA

pankaj@cs.duke.edu

\section{Boris Aronov}

Department of Computer Science and Engineering, Tandon School of Engineering, New York University, Brooklyn, NY 11201, USA

boris.aronov@nyu.edu

\section{Esther Ezra}

Department of Computer Science, Bar-Ilan University, Ramat Gan, Israel School of Math, Georgia Institute of Technology, Atlanta, Georgia 30332, USA eezra3@math.gatech.edu

\section{Joshua Zahl}

Department of Mathematics, University of British Columbia, Vancouver, BC V6T 1Z2, Canada jzahl@math.ubc.ca

\begin{abstract}
In 2015 , Guth proved that if $\mathcal{S}$ is a collection of $n g$-dimensional semi-algebraic sets in $\mathbb{R}^{d}$ and if $D \geq 1$ is an integer, then there is a $d$-variate polynomial $P$ of degree at most $D$ so that each connected component of $\mathbb{R}^{d} \backslash Z(P)$ intersects $O\left(n / D^{d-g}\right)$ sets from $\mathcal{S}$. Such a polynomial is called a generalized partitioning polynomial. We present a randomized algorithm that computes such polynomials efficiently - the expected running time of our algorithm is linear in $|\mathcal{S}|$. Our approach exploits the technique of quantifier elimination combined with that of $\varepsilon$-samples.

We present four applications of our result. The first is a data structure for answering pointenclosure queries among a family of semi-algebraic sets in $\mathbb{R}^{d}$ in $O(\log n)$ time, with storage complexity and expected preprocessing time of $O\left(n^{d+\varepsilon}\right)$. The second is a data structure for answering range search queries with semi-algebraic ranges in $O(\log n)$ time, with $O\left(n^{t+\varepsilon}\right)$ storage and expected preprocessing time, where $t>0$ is an integer that depends on $d$ and the description complexity of the ranges. The third is a data structure for answering vertical ray-shooting queries among semi-algebraic sets in $\mathbb{R}^{d}$ in $O\left(\log ^{2} n\right)$ time, with $O\left(n^{d+\varepsilon}\right)$ storage and expected preprocessing time. The fourth is an efficient algorithm for cutting algebraic planar curves into pseudo-segments.
\end{abstract}

2012 ACM Subject Classification Mathematics of computing $\rightarrow$ Combinatorial algorithms; Theory of computation $\rightarrow$ Randomness, geometry and discrete structures

Keywords and phrases Polynomial partitioning, quantifier elimination, semi-algebraic range spaces, $\varepsilon$-samples

Digital Object Identifier 10.4230/LIPIcs.SoCG.2019.5

Related Version https://arxiv.org/abs/1812.10269

Funding Pankaj K. Agarwal: P. Agarwal was supported by NSF under grants CCF-15-13816, CCF15-46392, and IIS-14-08846, by an ARO grant W911NF-15-1-0408, and by BSF Grant 2012/229 from the U.S.-Israel Binational Science Foundation.

Boris Aronov: B. Aronov was supported by NSF grants CCF-12-18791 and CCF-15-40656, and by grant 2014/170 from the US-Israel Binational Science Foundation.

Esther Ezra: E. Ezra was supported by NSF CAREER under grant CCF:AF 1553354 and by Grant $824 / 17$ from the Israel Science Foundation.

Joshua Zahl: J. Zahl was supported by an NSERC Discovery grant.

(c) (i) ( Pankaj K. Agarwal, Boris Aronov, Esther Ezra, and Joshua Zahl;

cc. licensed under Creative Commons License CC-BY

35th International Symposium on Computational Geometry (SoCG 2019).

Editors: Gill Barequet and Yusu Wang; Article No. 5; pp. 5:1-5:14

Leibniz International Proceedings in Informatics 


\section{Introduction}

In 2015, Guth [11] proved that if $\mathcal{S}$ is a collection of $n g$-dimensional semi-algebraic sets ${ }^{1}$ in $\mathbb{R}^{d}$ and if $D \geq 1$ is an integer, then there is a $d$-variate polynomial $P$ of degree at most $D$ so that each connected component of $\mathbb{R}^{d} \backslash Z(P)$ intersects $O\left(n / D^{d-g}\right)$ sets from $\mathcal{S}{ }^{2}$, where the implicit constant in the $O(\cdot)$ notation depends on $d$ and on the degree and number of polynomials required to define each semi-algebraic set. We refer to such a polynomial $P$ as a generalized partitioning polynomial. Guth's proof established the existence of a generalized partitioning polynomial, but it did not offer an efficient algorithm to compute such a polynomial for a given collection of semi-algebraic sets. In this paper we study the problem of computing a generalized partitioning polynomial efficiently and present a few applications of such an algorithm.

Related work. In 2010, Guth and Katz [12] resolved the Erdös distinct distances problem in the plane. A major ingredient in their proof was a partitioning theorem for points in $\mathbb{R}^{d}$. Specifically, they proved that given a set of $n$ points in $\mathbb{R}^{d}$ and an integer $D \geq 1$, there is a $d$-variate partitioning polynomial $P$ of degree at most $D$ so that each connected component of $\mathbb{R}^{d} \backslash Z(P)$ contains $O\left(n / D^{d}\right)$ points from the set. Their polynomial partitioning theorem led to a flurry of new results in combinatorial and incidence geometry, harmonic analysis, and theoretical computer science.

The Guth-Katz result established the existence of a partitioning polynomial, but it did not provide an effecient algorithm to compute such a polynomial. In [2], Agarwal, Matoušek, and Sharir developed an efficient algorithm to compute partitioning polynomials, matching the degree bound obtained in [12] up to a constant factor. They used this algorithm to construct a linear-size data structure that can answer semialgebraic range queries amid a set of $n$ points in $\mathbb{R}^{d}$ in time $O\left(n^{1-1 / d}\right.$ polylog $\left.(n)\right)$, which is near optimal.

A major open question in geometric searching is whether a semialgebraic range query can be answered in $O(\log n)$ time using a data structure of size roughly $n^{d}$; such a data structure is known only in very special cases, e.g., when query ranges are simplices [3]. If $t$ is the number of (real valued) parameters needed to represent query ranges, then the best known data structure for semialgebraic range searching uses $O\left(n^{t+\varepsilon}\right)$ space for $t \leq 4$ and $O\left(n^{2 t-4+\varepsilon}\right)$ space for $t>4[3]$. As we show below, an efficient algorithm for computing a generalized partitioning polynomial leads to a semialgebraic range searching data structure with $O(\log n)$ query time and $O\left(n^{t+\varepsilon}\right)$ space.

In [4], the last three authors developed an efficient algorithm for constructing a partition of $\mathbb{R}^{3}$ adapted to a set of space curves. This partition is not given by a partitioning polynomial, but it shares many of the same properties. For other settings, however, no effective method is known for computing a partitioning polynomial.

Our results. Our main result is an efficient algorithm for computing a generalized partitioning polynomial for a family of semi-algebraic sets (Theorem 11): Given a set $\mathcal{S}$ of $n$ semi-algebraic sets in $\mathbb{R}^{d}$, each of complexity at most $b$ for some constant $b>0$ (see Section 2 for the definition of the complexity of a semi-algebraic set), our algorithm computes a generalized partitioning polynomial of given degree $D$ in expected time $O\left(n e^{\operatorname{Poly}(D)}\right)$.

1 Roughly speaking, a semi-algebraic set in $\mathbb{R}^{d}$ is the set of points in $\mathbb{R}^{d}$ that satisfy a Boolean formula over a set of polynomial inequalities. See [8, Chapter 2] for formal definitions of a semialgebraic set and its dimension.

2 Guth stated his result for the special case where the semi-algebraic sets are real algebraic varieties, but his proof in fact holds in the more general setting of semi-algebraic sets. 
In Section 4 we present four applications of our algorithm:

(i) Let $\mathcal{S}$ be a family of $n$ semi-algebraic sets in $\mathbb{R}^{d}$, each of complexity at most $b$ for some constant $b>0$. Each set in $\mathcal{S}$ is assigned a weight that belongs to a semigroup. We present a data structure of size $O\left(n^{d+\varepsilon}\right)$, for any constant $\varepsilon>0$, that can compute, in $O(\log n)$ time, the cumulative weight of the sets in $\mathcal{S}$ containing a query point. We refer to the latter as a point-enclosure query. The data structure can be constructed in $O\left(n^{d+\varepsilon}\right)$ randomized expected time. This is a significant improvement over the best known data structure by Koltun [14], for $d>4$, that uses $O\left(n^{2 d-4+\varepsilon}\right)$ space.

(ii) Let $P$ be a set of $n$ points in $\mathbb{R}^{d}$, each of which is assigned a weight in a semigroup, and let $\mathcal{R}$ be a (possibly infinite) family of semi-algebraic sets in $\mathbb{R}^{d}$. Suppose that there exists a positive integer $t$ and an injection $f: \mathcal{R} \rightarrow \mathbb{R}^{t}$ so that for each $p \in P$, the set $f(\{R \in \mathcal{R} \mid p \in R\})$ is a semi-algebraic set in $\mathbb{R}^{t}$ of complexity at most $b$. We can construct in $O\left(n^{t+\varepsilon}\right)$ randomized expected time a data structure of size $O\left(n^{t+\varepsilon}\right)$, for any constant $\varepsilon>0$, that can compute in $O(\log n)$ time the cumulative weight of $P \cap \gamma$ for a query range $\gamma \in \mathcal{R}$.

(iii) Given a family $\mathcal{S}$ of $n$ semi-algebraic sets in $\mathbb{R}^{d}$, we present a data structure of size $O\left(n^{d+\varepsilon}\right)$, for any constant $\varepsilon>0$, that can answer vertical ray shooting queries in $O\left(\log ^{2} n\right)$ time. The data structure can be constructed in $O\left(n^{d+\varepsilon}\right)$ randomized expected time.

(iv) Finally, we follow the technique of Sharir and Zahl [15] to cut $n$ algebraic planar curves into a collection of $O\left(n^{3 / 2+\varepsilon}\right)$ pseudo-segments (that is, a collection of Jordan arcs, each pair of which intersects at most once), where the constant of proportionality depends on the degree of the curves. By exploiting Theorem 11, we show that this collection can be constructed in comparable time bound. We comment that applying the algorithm in the previous work of Aronov et al. [4] results in the same asymptotic bound on the number of pseudo-segments, however, its running time is quadratic in the number of curves. Thus our algorithm yields a considerable improvement in the running time.

Throughout this paper we assume that $d, b$, and $\varepsilon$ are constants. Whenever big-O notation is used, the implicit constant may depend on $d, b$, and $\varepsilon$.

\section{Preliminaries}

In what follows, the complexity of a semi-algebraic set $S$ in $\mathbb{R}^{d}$ is the minimum value $b$ so that $S$ can be represented as the set of points $x \in \mathbb{R}^{d}$ satisfying a Boolean formula with at most $b$ atoms of the form $P(x)<0$ or $P(x)=0$, with each $P$ being a $d$-variate polynomial of degree at most $b$.

Our analysis makes extensive use of concepts and results from real algebraic geometry and random sampling. We review them below.

\subsection{Polynomials, partitioning, and quantifier elimination}

Sign conditions. Let $\mathbb{R}\left[x_{1}, \ldots, x_{d}\right]$ denote the set of all $d$-variate polynomials with real coefficients. For $P_{1}, \ldots, P_{\ell} \in \mathbb{R}\left[x_{1}, \ldots, x_{d}\right]$, a sign condition on $\left(P_{1}, \ldots, P_{\ell}\right)$ is an element of $\{-1,0,1\}^{\ell}$. A strict sign condition on $\left(P_{1}, \ldots, P_{\ell}\right)$ is an element of $\{-1,1\}^{\ell}$. A sign condition $\left(\nu_{1}, \ldots, \nu_{\ell}\right) \in\{-1,0,1\}^{\ell}$ is realizable if the set

$$
\left\{x \in \mathbb{R}^{d} \mid \operatorname{sign}\left(P_{j}(x)\right)=\nu_{j} \text { for each } j=1, \ldots, \ell\right\}
$$


is non-empty. A realizable strict sign condition is defined analogously. The set (1) is called the realization of the sign condition. The set of realizations of sign conditions (resp., realizations of strict sign conditions) corresponding to the tuple $\left(P_{1}, \ldots, P_{\ell}\right)$ is the collection of all non-empty sets of the above form. These sets are pairwise disjoint and partition $\mathbb{R}^{d}$, by definition.

While a tuple of $\ell$ polynomials has $3^{\ell}$ sign conditions and $2^{\ell}$ strict sign conditions, Milnor and Thom (see, e.g., $[6,10]$ ) showed that any $\ell$ polynomials in $\mathbb{R}\left[x_{1}, \ldots, x_{d}\right]$ of degree at most $s$ (with $2 \leq d \leq \ell$ ) have at most $(50 s \ell / d)^{d}$ realizable sign conditions.

Polynomials and partitioning. The set of polynomials in $\mathbb{R}\left[x_{1}, \ldots, x_{d}\right]$ of degree at most $b$

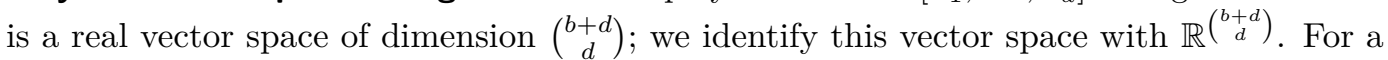
point $q \in \mathbb{R}^{\left(\begin{array}{c}b+d \\ d\end{array}\right)}$, let $P_{q} \in \mathbb{R}\left[x_{1}, \ldots, x_{d}\right]$ be the corresponding polynomial of degree at most $b$.

- Remark 1. Consider the polynomial $Q(q, x) \in \mathbb{R}\left[q_{1}, \ldots, q_{\left(\begin{array}{c}b+d \\ d\end{array}\right)}, x_{1}, \ldots, x_{d}\right]$ given by $Q(q, x):=P_{q}(x)$. Since we can write $Q(q, x)=\sum_{i=1}^{\left(\begin{array}{c}b+d \\ d\end{array}\right)} q_{i} H_{i}(x)$, where $H_{i}$ is a monomial of degree at most $b, Q$ has degree $b+1$.

For each positive integer $j$, let $D_{j}$ be the smallest positive integer so that $\left(\begin{array}{c}D_{j}+d \\ d\end{array}\right)>2^{j-1}$; we have $D_{j}=O\left(2^{j / d}\right)$. Let $k$ be a positive integer. For each $j=1, \ldots, k$, pick a $2^{j-1}$ -

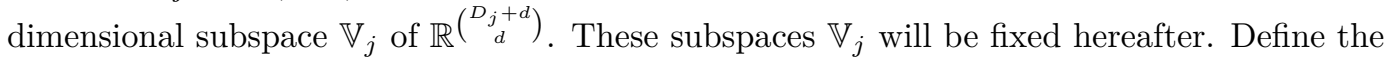
product space

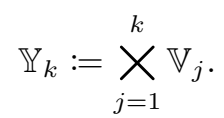

We identify each point $y=\left(y_{1}, \ldots, y_{k}\right) \in \mathbb{Y}_{k}$, where $y_{j} \in \mathbb{V}_{j}$, with a $k$-tuple of polynomials $\mathbf{P}_{y}=\left(P_{y_{1}}, \ldots, P_{y_{k}}\right)$. For each $j=1, \ldots, k, \operatorname{deg}\left(P_{j}\right)=O\left(2^{j / d}\right)$ and thus $\operatorname{deg}\left(\prod_{j=1}^{k} P_{j}\right)=$ $O\left(2^{k / d}\right)$.

Let $\mathbf{P}_{y} \in \mathbb{Y}_{k}$, let $\mathcal{S}$ be a collection of semi-algebraic sets in $\mathbb{R}^{d}$, and let $\alpha \geq 1$. We say that $\mathbf{P}_{y}$ is a $(k, \alpha)$-partitioning tuple for $\mathcal{S}$ if $\mathbf{P}_{y}$ has exactly $2^{k}$ realizable strict sign conditions and the realization of each of them intersects at most $|\mathcal{S}| / \alpha$ sets from $\mathcal{S} .^{3}$ Guth [11] proved that, for an appropriate choice of $\alpha$, a $(k, \alpha)$-partitioning tuple is guaranteed to exist:

- Proposition 2 (Generalized Polynomial Partitioning [11]). Let $\mathcal{S}$ be a family of semi-algebraic sets in $\mathbb{R}^{d}$, each of dimension at most $g$ and complexity at most $b$. For each $k \geq 1$, there exists a $(k, \alpha)$-partitioning tuple for $\mathcal{S}$, with $\alpha=\Omega_{b, d}\left(2^{k(1-g / d)}\right)$.

We also recall Theorem 2.16 from [7]:

- Proposition 3. Let $\mathcal{P}$ be a set of $s$ polynomials in $\mathbb{R}\left[x_{1}, \ldots, x_{d}\right]$ of degree at most $t$. Then there is an algorithm that computes a set of points meeting every connected component of every realizable sign condition on $\mathcal{P}$ in time $O\left(s^{d} t^{O(d)}\right)$. There is also an algorithm providing the list of signs of all the polynomials of $\mathcal{P}$ at each of these points in time $O\left(s^{d+1} t^{O(d)}\right)$.

3 As in [11], we work with a $k$-tuple of polynomials instead of a single polynomial so that we can bound the number of sets intersected by the realization of a sign condition rather than by a connected component of a realization. 
Singly exponential quantifier elimination. Let $h$ and $\ell$ be non-negative integers and let $\mathcal{P}=\left\{P_{1}, \ldots, P_{s}\right\} \subset \mathbb{R}\left[x_{1}, \ldots, x_{h}, y_{1}, \ldots, y_{\ell}\right]$. Let $\Phi(y)$ be a first-order formula given by

$$
\left(\exists x_{1}, \ldots, x_{h}\right) F\left(P_{1}(x, y), \ldots, P_{s}(x, y)\right),
$$

where $y=\left(y_{1}, \ldots, y_{\ell}\right)$ is a block of $\ell$ free variables; $x$ is a block of $h$ variables, and $F\left(P_{1}, \ldots, P_{s}\right)$ is a quantifier-free Boolean formula with atomic predicates of the form $\operatorname{sign}\left(P_{i}\left(x_{1}, \ldots, x_{h}, y\right)\right)=\sigma$, with $\sigma \in\{-1,0,1\}$.

The Tarski-Seidenberg theorem states that the set of points $y \in \mathbb{R}^{\ell}$ satisfying the formula $\Phi(y)$ is semi-algebraic. The next proposition is a quantitative version of this result that bounds the number and degree of the polynomial equalities and inequalities needed to describe the set of points satisfying $\Phi(y)$. This proposition is known as a "singly exponential quantifier elimination," and its more general form (where $\Phi(y)$ may contain a mix of $\forall$ and $\exists$ quantifiers) can be found in [7, Theorem 2.27].

- Proposition 4. Let $\mathcal{P}$ be a set of at most s polynomials, each of degree at most $t$ in $h+\ell$ real variables. Given a formula $\Phi(y)$ of the form (3), there exists an equivalent quantifier-free formula

$$
\Psi(y)=\bigvee_{i=1}^{I} \bigwedge_{j=1}^{J_{i}}\left(\bigvee_{n=1}^{N_{i, j}} \operatorname{sign}\left(P_{i j n}(y)\right)=\sigma_{i j n}\right),
$$

where $P_{i j n}$ are polynomials in the variables $y, \sigma_{i j n} \in\{-1,0,1\}$,

$$
\begin{aligned}
I & \leq s^{(h+1)(\ell+1)} t^{O(h \cdot \ell)}, \\
J_{i} & \leq s^{(h+1)} t^{O(h)}, \\
N_{i j} & \leq t^{O(h)},
\end{aligned}
$$

and the degrees of the polynomials $P_{i j n}(y)$ are bounded by $t^{O(h)}$. Moreover, there is an algorithm to compute $\Psi(Y)$ in time $s^{(h+1)(\ell+1)} t^{O(h \cdot \ell)}$.

\subsection{Range spaces, VC dimension, and $\varepsilon$-samples}

We first recall several standard definitions and results from [13, Chapter 5]. A range space is a pair $\Sigma=(X, \mathcal{R})$, where $X$ is a set and $\mathcal{R}$ is a collection of subsets of $X$. Let $(X, \mathcal{R})$ be a range space and let $A \subset X$ be a set. We define the restriction of $\Sigma$ to $A$, denoted by $\Sigma_{A}$ to be $\left(A, \mathcal{R}_{A}\right)$, where $\mathcal{R}_{A}:=\{R \cap A \mid R \in \mathcal{R}\}$. If $A$ is finite, then $\left|\mathcal{R}_{A}\right| \leq 2^{|A|}$. If equality holds, then we say $A$ is shattered. We define the shatter function by $\pi_{\mathcal{R}}(z):=\max _{|A|=z}\left|\mathcal{R}_{A}\right|$. The $V C$ dimension of $\Sigma$ is the largest cardinality of a set shattered by $\mathcal{R}$. If arbitrarily large finite subsets can be shattered, we say that the $\mathrm{VC}$ dimension of $\Sigma$ is infinite.

Let $\Sigma$ be a range space, $A$ a finite subset of $X$, and $0 \leq \varepsilon \leq 1$. A set $B \subset A$ is an $\varepsilon$-sample (also known as $\varepsilon$-approximation) of $\Sigma_{A}$ if

$$
\left|\frac{|A \cap R|}{|A|}-\frac{|B \cap R|}{|B|}\right| \leq \varepsilon \quad \forall R \in \mathcal{R} .
$$

The following classical theorem of Vapnik and Chervonenkis [16] guarantees that, if the VC-dimension of $\Sigma$ is finite, then for each positive $\varepsilon>0$, a sufficiently large random sample of $A$ is likely to be an $\varepsilon$-sample. ${ }^{4}$

\footnotetext{
4 The stated bound is not the strongest possible (see, e.g., [13, Chapter 7] for an improved bound), but is sufficient for our purposes.
} 
- Proposition 5 ( $\varepsilon$-Sample Theorem). Let $\Sigma=(X, \mathcal{R})$ be a range space of $V C$ dimension at most $d$ and let $A \subset X$ be finite. Let $0<\varepsilon, \delta<1$. Then a random subset $B \subset A$ of cardinality $\frac{8 d}{\varepsilon^{2}} \log \frac{1}{\varepsilon \delta}$ is an $\varepsilon$-sample for $\Sigma_{A}$ with probability at least $1-\delta$.

- Proposition $6([10,13])$. Let $\Sigma=(X, \mathcal{R})$ be a range space whose shatter function $\pi_{\mathcal{R}}(z)$ satisfies the bound $\pi_{\mathcal{R}}(z) \leq C z^{\rho}$, for all positive integers $z$, where $\rho>0$ is a real parameter. Then $\Sigma$ has $V C$ dimension at most $4 \rho \log (C \rho)$.

The following theorem can be proven by an argument closely following that in the proof of Corollary 2.3 from [10]; see [1] for details.

- Theorem 7. Let $Z \subset \mathbb{R}^{d} \times \mathbb{R}^{\ell}$ be a semi-algebraic set of complexity $b$. For each $y \in \mathbb{R}^{\ell}$, define $R_{y}=\left\{x \in \mathbb{R}^{d} \mid(x, y) \in Z\right\}$. Then the range space $\left(\mathbb{R}^{d},\left\{R_{y} \mid y \in \mathbb{R}^{\ell}\right\}\right)$ has VC dimension at most $200 \ell^{2} \log b$.

\section{Computing Generalized Polynomial Partition}

In this section we obtain the main result of the paper: given a collection $\mathcal{S}$ of semi-algebraic sets in $\mathbb{R}^{d}$, each of dimension at most $g$ and complexity at most $b$, a $\left(k, \Omega_{b, d}\left(D^{d-g}\right)\right)$-partitioning tuple for $\mathcal{S}$ can be computed efficiently. We obtain this result in several steps. Given a semialgebraic set $S$, a sign condition $\sigma \in\{-1,+1\}^{k}$ and a real value $b>0$, we first show that the set of $k$-tuples of degree- $b$ polynomials whose realization of $\sigma$ intersects $S$ is a semialgebraic set. This in turn implies that, if $S_{1}, \ldots, S_{n}$ are semi-algebraic sets and if $m \leq n$, then the set of $k$-tuples of degree- $b$ polynomials whose realization intersects at most $m$ of the sets $S_{1}, \ldots, S_{n}$ is semi-algebraic. We use a quantifier-elimination algorithm to find a desired $k$-tuple. Unfortunately, the running time of the algorithm is exponential in $n$. We reduce the running time of the algorithm by using a random sampling technique - we show that it suffices to compute a partitioning tuple with respect to a small-size random subset of $\mathcal{S}$.

\subsection{The parameter space of semi-algebraic sets}

Fix positive integers $b, d, g$, and $k$, and let $D=2^{k / d}$. Hereafter we assume that $D=\Omega\left(2^{b}\right)$, which can be enforced by choosing $k$ sufficiently large.

As above, let $\mathcal{S}$ be a family of semi-algebraic sets in $\mathbb{R}^{d}$, each of dimension at most $g$ and complexity at most $b$. Let $G:\{0,1\}^{b} \rightarrow\{0,1\}$ be a Boolean function. Let $\mathbb{X}=\left(\mathbb{R}^{\left(\begin{array}{c}b+d \\ d\end{array}\right)}\right)^{b}$. We identify a point $x=\left(q_{1}, \ldots, q_{b}\right) \in \mathbb{X}$ with the semi-algebraic set

$$
Z_{x, G}=\left\{v \in \mathbb{R}^{d} \mid G\left(P_{q_{1}}(v) \geq 0, \ldots, P_{q_{b}}(v) \geq 0\right)=1\right\} \subset \mathbb{R}^{d} .
$$

Observe that each semi-algebraic set in $\mathcal{S}$ is of the form $Z_{x, G}$ for some choice of $x \in \mathbb{X}$ and a Boolean function $G$. Let $\mathbb{Y}=\mathbb{Y}_{k}$. For each $y \in \mathbb{Y}$, define $S_{y}:=\left\{u \in \mathbb{R}^{d} \mid P_{1}(u)>\right.$ $\left.0, \ldots, P_{k}(u)>0\right\}$, where $\left(P_{1}, \ldots, P_{k}\right)$ is the tuple associated with $y$. Define

$$
W_{G}:=\left\{(x, y) \in \mathbb{X} \times \mathbb{Y} \mid Z_{x, G} \cap S_{y} \neq \emptyset\right\} .
$$

- Theorem 8. The set $W_{G}$ is semi-algebraic; it is defined by $O\left(e^{\operatorname{Poly}(D)}\right)$ polynomials, each of degree $D^{O(d)}$.

Proof. Define $V=\left\{(x, y, v) \in \mathbb{X} \times \mathbb{Y} \times \mathbb{R}^{d} \mid v \in Z_{x, G} \cap S_{y}\right\}$. The condition $v \in Z_{x, G}$ is a Boolean condition on $b$ polynomial inequalities. By Remark 1, each of these polynomials has degree at most $b+1$. Similarly, the condition $v \in S_{y}$ consists of $k$ polynomial inequalities, each 
of degree at most $D+1$. This means that there exists a set of polynomials $Q=\left\{Q_{1}, \ldots, Q_{b+k}\right\}$ of degree $b+D+1$ in the variables $x, y, v$, and a Boolean function $F\left(z_{1}, \ldots, z_{b+k}\right)$ so that

$$
V=\left\{(x, y, v) \in \mathbb{X} \times \mathbb{Y} \times \mathbb{R}^{d} \mid F\left(Q_{1}(x, y, v) \geq 0, \ldots, Q_{b+k}(z, y, v) \geq 0\right)=1\right\} .
$$

With the above definitions

$$
W_{G}=\left\{(x, y) \mid \exists\left(v_{1}, \ldots, v_{d}\right): F\left(Q_{1}(x, y, v), \ldots, Q_{b+k}(x, y, v)\right)=1\right\}
$$

We now apply Proposition 4 . We have a set $\mathcal{Q}$ of $s=b+k$ polynomials, each of degree at most $t=b+D+1$. The variables $h$ and $\ell$ from the hypothesis of Proposition 4 are set to $h=d$ and $\ell=O\left(\left(\begin{array}{c}b+d \\ d\end{array}\right)^{b}+D^{d}\right)=\operatorname{Poly}(D)$; recall that $D$ is sufficiently larger than $b$, and thus $\ell$ is a suitably chosen polynomial function of $D$. With these assignments, Proposition 4 says that $W_{G}$ can be expressed as a quantifier-free formula of the form

$$
\bigvee_{i=1}^{I} \bigwedge_{j=1}^{J_{i}}\left(\bigvee_{n=1}^{N_{i, j}} \operatorname{sign}\left(P_{i j n}(x, y)\right)=\sigma_{i j n}\right)
$$

where $P_{i j n}$ are polynomials in the variables $(x, y), \sigma_{i j n} \in\{-1,0,1\}$,

$$
\begin{aligned}
I & \leq(b+k)^{\operatorname{Poly}(D)}(b+D)^{\operatorname{Poly}(D)}=O\left(e^{\operatorname{Poly}(D)}\right), \\
J_{i} & \leq(b+k)^{d+1}(b+D)^{O(d)}=O\left(D^{O(d)}\right), \\
N_{i j} & \leq(b+D)^{O(d)}=O\left(D^{O(d)}\right),
\end{aligned}
$$

where the degrees of the polynomials $P_{i j n}(y)$ are bounded by $(b+D)^{O(d)}=D^{O(d)}$.

Summarizing, the quantifier-free formula (6) for $W_{G}$ is a Boolean combination of $O\left(e^{\operatorname{Poly}(D)}\right)$ polynomial inequalities, each of degree $D^{O(d)}$, as claimed.

\subsection{A singly-exponential algorithm}

In this section, we discuss how to compute a $(k, \alpha)$-partitioning tuple (for an appropriate value of $\alpha$ ) for a small number $m$ of semi-algebraic sets.

- Theorem 9. Let $\mathcal{S}$ be a family of $m$ semi-algebraic sets in $\mathbb{R}^{d}$, each of dimension at most $g$ and complexity at most $b$. Let $1 \leq k \leq \log m$ and let $D=2^{k / d}$. Then $a\left(k, \Omega_{b, d}\left(D^{d-g}\right)\right)$ partitioning tuple for $\mathcal{S}$ can be computed in $O\left(e^{\mathrm{Poly}(m)}\right)$ time.

Proof. Set $\mathbb{Y}=\mathbb{Y}_{k}$. As above, we identify points in $\mathbb{Y}$ with $k$ tuples $\left(P_{1}, \ldots, P_{k}\right)$ of polynomials. The argument in Theorem 8 , as well as the fact that the class of semi-algebraic sets is closed under the operation of taking a projection, show that for each $S \in \mathcal{S}$ and each $\sigma \in\{-1,1\}^{k}$

$$
I_{S, \sigma}:=\left\{y \in \mathbb{Y} \mid S \cap\left\{\sigma_{1} P_{1}>0, \sigma_{2} P_{2}>0, \ldots, \sigma_{k} P_{k}>0\right\} \neq \emptyset\right\}
$$

is a semi-algebraic set in $\mathbb{Y}$ that can be expressed as a Boolean combination of $O\left(e^{\operatorname{Poly}(D)}\right)$ polynomials, each of degree $D^{O(d)}$. Moreover, it can be computed in time $O\left(e^{\operatorname{Poly}(D)}\right)$ (see once again Proposition 4).

Let $C_{b, d}$ be a constant to be specified later (the constant will depend only on $b$ and $d$ ) and let $N=C_{b, d}|\mathcal{S}| D^{g-d}+1$; observe that $N=O(m)$. For each $\sigma \in\{-1,1\}^{k}$ and for each set $\mathcal{S}^{\prime} \subset \mathcal{S}$ of cardinality $\left|\mathcal{S}^{\prime}\right| \geq N$, the set $\left\{y \in \mathbb{Y} \mid y \in I_{S, \sigma}\right.$ for every $\left.S \in \mathcal{S}^{\prime}\right\}$ is a semi-algebraic 
set in $\mathbb{Y}$ that can be expressed as a Boolean combination of $O\left(N^{\prime} e^{\operatorname{Poly}(D)}\right)=O\left(m e^{\mathrm{Poly}(D)}\right)$ polynomials, each of degree $D^{O(d)}$, where $N^{\prime}=\left|\mathcal{S}^{\prime}\right|$. Therefore

$$
\mathcal{K}:=\bigcup_{\substack{S^{\prime} \subset \mathcal{S} \\\left|\mathcal{S}^{\prime}\right| \geq N}}\left\{y \in \mathbb{Y} \mid y \in I_{S, \sigma} \text { for every } S \in \mathcal{S}^{\prime}\right\}
$$

is a semi-algebraic set in $\mathbb{Y}$ that can be expressed as a Boolean combination of

$$
\sum_{\substack{S^{\prime} \subset \mathcal{S} \\
\left|\mathcal{S}^{\prime}\right| \geq N}} O\left(\left(\begin{array}{c}
m \\
\left|S^{\prime}\right|
\end{array}\right) m e^{\operatorname{Poly}(D)}\right)=O\left(e^{m+\operatorname{Poly}(D)}\right)=O\left(e^{\operatorname{Poly}(m)}\right)
$$

polynomials, each of degree $D^{O(d)}$. This and the fact that the class of semi-algebraic sets is closed under the operation of taking complement imply that

$$
\operatorname{Good}(\sigma):=\mathbb{Y} \backslash \mathcal{K}=\left\{y \in \mathbb{Y} \mid y \in I_{S, \sigma} \text { for at most } C_{b, d}|\mathcal{S}| D^{g-d} \text { sets } S \in \mathcal{S}\right\}
$$

is a semi-algebraic set in $\mathbb{Y}$ that can be expressed as a Boolean combination of $O\left(e^{\mathrm{Poly}(m)}\right)$ polynomials, each of degree $D^{O(d)}$. This means that the set

$$
\bigcap_{\sigma \in\{-1,1\}^{k}} \operatorname{Good}(\sigma)
$$

is a semi-algebraic set in $\mathbb{Y}$ that can be expressed as a Boolean combination of $O\left(e^{\operatorname{Poly}(m)}\right)$ polynomials, each of degree $D^{O(d)}$. Recall that by assumption $1 \leq k \leq \log m$ and $D=2^{k / d}$. It thus follows that the degree is bounded by $\operatorname{Poly}(m)$. Similarly, the dimension of the space $\mathbb{Y}$ is bounded by $\operatorname{Poly}(m)$ as well.

Proposition 2 guarantees that if $C_{b, d}$ is selected sufficiently large, then the set (9) is non-empty. By Proposition 3, it is possible to locate a point in this set in $O\left(e^{\operatorname{Poly}(m)}\right)$ time, concluding the proof of the theorem.

\subsection{Speeding up the algorithm using $\varepsilon$-sampling}

In this section we first state the following lemma, whose proof is omitted (see the full version of this paper [1] for the details):

- Lemma 10. For every choice of positive integers $b$ and $d$, there is a constant $C=C(b, d)$ so that the following holds. Let $C_{0}$ be a positive integer. Let $\mathcal{S}$ be a finite collection of semi-algebraic sets in $\mathbb{R}^{d}$, each of dimension at most $g$ and complexity at most $b$. Let $k$ be a positive integer and let $D=2^{k / d}$. Let $B \subset \mathcal{S}$ be a randomly chosen subset of $\mathcal{S}$ of cardinality at least $C D^{C}$ and let $\left(P_{1}, \ldots, P_{k}\right)$ be a $\left(k, \frac{D^{d-g}}{C_{0}}\right)$-partitioning tuple for $B$. Then with probability at least $1 / 2$, each of the $D^{d}$ realizable sign conditions of $\left(P_{1}, \ldots, P_{k}\right)$ intersects $O\left(|\mathcal{S}| C_{0} D^{g-d}\right)$ elements from $\mathcal{S}$.

Note that Lemma 10 states that it is sufficient to consider a random subset $B$ of size polynomial in $D$ in order to obtain an appropriate partitioning tuple for the entire collection $\mathcal{S}$, with reasonable probability.

We next proceed as follows. We select a random sample of $\mathcal{S}$ of cardinality $C D^{C}$ and use Theorem 9 to compute the corresponding partitioning tuple $\left(P_{1}, \ldots, P_{k}\right)$. This takes $O\left(e^{\text {Poly }(D)}\right)$ time. By Lemma 10 , this tuple will be a $\left(k, \Omega_{b, d}\left(D^{d-g}\right)\right)$-partitioning tuple for $\mathcal{S}$ with probability at least $1 / 2$. We can verify whether the partitioning tuple works in 
$O(|\mathcal{S}| \operatorname{Poly}(D))$ time. If the tuple does not produce the appropriate partition, we discard it and try again; the expected number of trials is at most 2. The verification step is done as follows. For each semi-algebraic set $S \in \mathcal{S}$ we compute the subset of sign conditions of $\left(P_{1}, \ldots, P_{k}\right)$, with which it has a non-empty intersection. To this end, we restrict each of the polynomials $P_{1}, \ldots, P_{k}$ to $S$ and apply Proposition 3 on this restricted collection, thereby obtaining a set of points meeting each connected component of each of the realizable sign conditions, as well as the corresponding list of signs of the restricted polynomials for each of these points. This is done in $O\left(D^{O(d)}\right)$ time for a single semi-algebraic set $S \in \mathcal{S}$, and overall $O\left(|\mathcal{S}| D^{O(d)}\right)$ time, over all sets. We refer the reader to [5] for further details concerning the complexity of the restriction of $P_{1}, \ldots, P_{k}$ to $S$. We have thus shown:

- Theorem 11. Let $\mathcal{S}$ be a finite collection of semi-algebraic sets in $\mathbb{R}^{d}$, each of which has dimension at most $g$ and complexity at most $b$. Let $k \geq 1$ and let $D=2^{k / d}$. Then a $\left(k, \Omega_{b, d}\left(D^{d-g}\right)\right)$-partitioning tuple for $\mathcal{S}$ can be computed in $O\left(|\mathcal{S}| \operatorname{Poly}(D)+e^{\operatorname{Poly}(D)}\right)$ expected time by a randomized algorithm.

\section{Applications}

In this section we describe a few applications of Theorem 11, namely, point-enclosure queries amid semi-algebraic sets, semi-algebraic range searching with logarithmic query time, vertical ray shooting amid semi-algebraic sets, and cutting algebraic curves into pseudo-segments.

\subsection{Point-enclosure queries}

Let $\mathcal{S}$ be a set of $n$ semi-algebraic sets in $\mathbb{R}^{d}$, each of complexity at most $b$. Each set $S$ is assigned a weight $w(S)$. We assume that the weights belong to a semigroup, i.e., subtractions are not allowed, and that the semigroup operation can be performed in constant time. We wish to preprocess $\mathcal{S}$ into a data structure so that the cumulative weight of the sets in $\mathcal{S}$ that contain a query point can be computed in $O(\log n)$ time; we refer to this query as point-enclosure query. Note that if the weight of each set is 1 and the semi-group operation is Boolean $\vee$, then the point-enclosure query becomes a union-membership query: determine whether the query point lies in $\bigcup \mathcal{S}$.

We follow a standard hierarchical partitioning scheme of space, e.g., as in [9, 3], but use Theorem 11 at each stage. Using this hierarchical partition, we construct a tree data structure $\mathcal{T}$ of depth $O(\log n)$, and a query is answered by following a path in $\mathcal{T}$. More precisely, we fix sufficiently large positive constants $D=D(b, d)$ and $n_{0}=n_{0}(D)$. If $n \leq n_{0}$ $\mathcal{T}$ consists of a single node that stores $\mathcal{S}$ itself. So assume that $n>n_{0}$. Using Theorem 11 , we construct a tuple $\mathcal{P}=\left(P_{1}, \ldots, P_{k}\right)$ of $d$-variate polynomials of degree at most $D$, which have $2^{k}=O\left(D^{d}\right)$ realizable sign conditions, each of which with a realization that meets the boundaries of at most $O(|\mathcal{S}| / D)$ sets of $\mathcal{S}$. For each realizable sign condition $\sigma$, let $\mathcal{S}_{\sigma} \subseteq \mathcal{S}$ be the family of sets whose boundaries meet the realization of $\sigma$, and let $\mathcal{S}_{\sigma}^{*} \subseteq \mathcal{S}$ be the family of sets that contain the realization of $\sigma$.

We compute $\mathcal{S}_{\sigma}, \mathcal{S}_{\sigma}^{*}$, and $W_{\sigma}=w\left(\mathcal{S}_{\sigma}^{*}\right)$, as follows: We first apply Proposition 3 to $\mathcal{P}$ to compute, in $O\left(D^{O(d)}\right)$ time, a representative point $\xi_{\sigma}$ in the realization of every realizable sign condition $\sigma$. We fix a set $S \in \mathcal{S}$, mark every realizable sign condition $\sigma$ that meets $\partial S$, and add $S$ to the set $\mathcal{S}_{\sigma}$. This step is similar to the one described in the proof of Theorem 11, that is, we restrict each of the polynomials $P_{1}, \ldots, P_{k}$ to the algebraic varieties representing the boundary of $S$ and apply Proposition 3 to this restricted collection. Each remaining sign condition $\sigma$ is either contained in $S$ or disjoint from it, which can be determined by testing whether its representing point $\xi_{\sigma}$ is contained in $S$. If the answer is yes, we add the weight $w(S)$ to $W_{\sigma}$. This task can be completed in overall $O\left(n D^{O(d)}\right)$ time over all sets of $\mathcal{S}$. 
We create the root $v$ of $\mathcal{T}$ and store the tuple $\mathcal{P}$ at $v$. We then create a child $z_{\sigma}$ for each realizable sign condition $\sigma$ and store $\sigma$ and $W_{\sigma}$ at $z_{\sigma}$. We recursively construct the data structure for each $\mathcal{S}_{\sigma}$ and attach it to $z_{\sigma}$ as its subtree. Since each node of $\mathcal{T}$ has degree at most $O\left(D^{d}\right)$ and the size of the subproblem reduces by a factor of $D$ at each level of the recursion, a standard analysis shows that the total size of the data structure is $O\left(n^{d+\varepsilon}\right)$, where $\varepsilon>0$ is a constant that can be made arbitrarily small by choosing $D$ and $n_{0}$ to be sufficiently large. Similarly, the expected preprocessing time is also $O\left(n^{d+\varepsilon}\right)$.

Given a query point $q \in \mathbb{R}^{d}$, we compute the cumulative weight of the sets containing $q$ by traversing a path in the tree in a top-down manner: We start from the root and maintain a partial weight $W$, which is initially set to 0 . At each node $v$, we find the sign condition $\sigma$ of the polynomial tuple at $v$ whose realization contains $q$, add $W_{\sigma}$ to $W$, and recursively query the child $z_{\sigma}$ of $v$. The total query time is $O(\log n)$, where the constant of proportionality depends on $D$ (and thus on $\varepsilon$ ). Putting everything together, we obtain the following:

- Theorem 12. Let $\mathcal{S}$ be a set of $n$ semi-algebraic sets in $\mathbb{R}^{d}$, each of complexity at most $b$ for some constant $b>0$, and let $w(S)$ be the weight of each set $S \in \mathcal{S}$ that belongs to a semigroup. Assuming that the semigroup operation can be performed in constant time, $\mathcal{S}$ can be preprocessed in $O\left(n^{d+\varepsilon}\right)$ randomized expected time into a data structure of size $O\left(n^{d+\varepsilon}\right)$, for any constant $\varepsilon>0$, so that the cumulative weight of the sets that contain a query point can be computed in $O(\log n)$ time.

\subsection{Range searching}

Next, we consider range searching with semi-algebraic sets: Let $P$ be a set of $n$ points in $\mathbb{R}^{d}$. Each point $p \in P$ is assigned a weight $w(p)$ that belongs to a semigroup. Again we assume that the semigroup operation takes constant time. We wish to preprocess $P$ so that, for a query range $\gamma$, represented as a semi-algebraic set in $\mathbb{R}^{d}$, the cumulative weight of $\gamma \cap P$ can be computed in $O(\log n)$ time. Here we assume that the query ranges (semi-algebraic sets) are parameterized as described in Section 3.1. That is, we have a fixed $b$-variate Boolean function $G$. A query range is represented as a point $x \in \mathbb{X}=\mathbb{R}^{t}$, for some $t \leq\left(\begin{array}{c}b+d \\ d\end{array}\right)^{b}$, and the underlying semi-algebraic set is $Z_{x, G}$. We refer to $t$ as the dimension of the query space, and to the range searching problem in which all query ranges are of the form $Z_{x, G}$ as $(G, t)$-semi-algebraic range searching.

For a point $p \in \mathbb{R}^{d}$, let $S_{p} \subseteq \mathbb{X}$ denote the set of semi-algebraic sets $Z_{x, G}$ that contain $p$, i.e., $S_{p}=\left\{x \in \mathbb{X} \mid p \in Z_{x, G}\right\}$. It can be checked that $S_{p}$ is a semi-algebraic set whose complexity depends only on $b, d$, and $G$. Let $\mathcal{S}=\left\{S_{p} \mid p \in P\right\}$. For a query range $Z_{x, G}$, we now wish to compute the cumulative weight of the sets in $\mathcal{S}$ that contain $x$. This can be done using Theorem 12. Putting everything together, we obtain the following:

- Theorem 13. Let $P$ be a set of $n$ points in $\mathbb{R}^{d}$, let $w(p)$ be the weight of $p \in P$ that belongs to a semigroup, and let $G$ be a fixed b-variate Boolean function for some constant $b>0$. Let $t \leq\left(\begin{array}{c}b+d \\ d\end{array}\right)$ be the dimension of the query space. Assuming that the semigroup operation can be performed in constant time, $P$ can be preprocessed in $O\left(n^{t+\varepsilon}\right)$ randomized expected time into a data structure of size $O\left(n^{t+\varepsilon}\right)$, for any constant $\varepsilon>0$, so that a $(G, t)$-semi-algebraic range query can be answered in $O(\log n)$ time.

- Remark. If $G$ is the conjunction of a set of $b$ polynomial inequalities, then the size of the data structure can be significantly improved by using a multi-level data structure, with a slight increase in the query time to $O\left(\log ^{b} n\right)$; see, e.g., [3]. Roughly speaking, the value of $t$ will now be the dimension of the parametric space of each polynomial defining the query semi-algebraic set, rather than the dimension of the parametric space of the entire semi-algebraic set (which is the conjunction of $b$ such polynomials). 


\subsection{Vertical ray shooting}

We next present an efficient data structure for answering vertical ray-shooting queries: Preprocess a collection $\mathcal{S}$ of $n$ semi-algebraic sets in $\mathbb{R}^{d}$, each of complexity at most $b$, into a data structure so that the first set of $\mathcal{S}$ hit by $\rho_{q}$, the ray emanating in the $\left(+x_{d}\right)$-direction from a query point $q$, can be reported quickly. If there is more than one such set, the query procedure returns one of them, arbitrarily.

The overall data structure. Our data structure for vertical ray shooting is similar to the one described in Section 4.1, except that we store an auxiliary data structure at each node of the tree to determine which of its children the query procedure should visit recursively.

Again we fix two constants $D$ and $n_{0}$. If $n \leq n_{0}$, the tree data structure $\mathcal{T}$ consists of a single node that stores $\mathcal{S}$. So assume that $n>n_{0}$. We compute a partitioning polynomial tuple $\mathcal{P}=\left(P_{1}, \ldots, P_{k}\right)$ for $\mathcal{S}$ of degree $D$. For each realizable sign condition $\sigma$, we compute the set $\mathcal{S}_{\sigma} \subseteq \mathcal{S}$ whose boundaries meet the realization of $\sigma$. We create the root node $v$ of $\mathcal{T}$ and create a child $z_{\sigma}$ for each realizable sign condition $\sigma$. We store two auxiliary data structures $\operatorname{DS}_{1}(v)$ and $\operatorname{DS}_{2}(v)$ at $v$, described below, each of which can be constructed in $O\left(n^{d+\varepsilon}\right)$ randomized expected time and requires $O\left(n^{d+\varepsilon}\right)$ space. Given a query point $q \in \mathbb{R}^{d}, \mathrm{DS}_{1}(v), \mathrm{DS}_{2}(v)$ together determine, in $O(\log n)$ time, the sign condition $\sigma$ whose realization contains the first intersection point of $\rho_{q}$ with a set of $\mathcal{S}$. We recursively construct the data structure for each subset $\mathcal{S}_{\sigma}$ and attach it to $z_{\sigma}$ as its subtree.

A standard analysis of multi-level data structures (see e.g. [3]) shows that the total size of $\mathcal{T}$ is $O\left(n^{d+\varepsilon}\right)$, for any constant $\varepsilon>0$, and that it can be constructed in $O\left(n^{d+\varepsilon}\right)$ randomized expected time.

For a query point $q \in \mathbb{R}^{d}$, the first set hit by $\rho_{q}$ can be computed by traversing a root-to-leaf path in $\mathcal{T}$. Suppose we are at a node $v$. If $v$ is a leaf, then we naively check all sets in $\mathcal{S}_{v}$ to find the first among them hit by $\rho_{q}$. Otherwise, we use the auxiliary data structures $\mathrm{DS}_{1}(v)$ and $\mathrm{DS}_{2}(v)$ to determine in $O(\log n)$ time the sign condition $\sigma$ whose realization contains the first intersection point of $\rho_{q}$ and a set of $\mathcal{S}$. We recursively visit the child $z_{\sigma}$ of $v$. Since the depth of $\mathcal{T}$ is $O(\log n)$, the total query time is $O\left(\log ^{2} n\right)$.

This completes the description of the overall algorithm. What remains is to describe the auxiliary data structures $\mathrm{DS}_{1}, \mathrm{DS}_{2}$.

Auxiliary data structures. Recall that the auxiliary data structures are used to determine the sign condition of $\mathcal{P}$ whose realization contains the first intersection point of a vertical ray with a set of $\mathcal{S}$. We first refine the realizations of sign conditions of $\mathcal{P}$ into "cylindrical" cells, as follows. Let $f=\prod_{i=1}^{k} P_{i}$; by construction, the degree of $f$ is $O(D)$. By Warren $[17$, Theorem 2], the number of connected components of $\mathbb{R}^{d} \backslash Z(f)$ is at most $O(D)^{d}$; from now on we refer to these components as cells. ${ }^{5}$ We refine the cells of $\mathbb{R}^{d} \backslash Z(f)$ using the so-called first-stage CAD (cylindrical algebraic decomposition); see, e.g., [6, Chapter 5] for a detailed overview of standard CAD. That is, this is a simplified version of CAD, presented in [2].

Roughly speaking, the first-stage CAD for $f$ is obtained by constructing a collection $\mathcal{G}$ of polynomials in the variables $x_{1}, \ldots, x_{d-1}$, whose zero sets contain the projection onto $\mathbb{R}^{d-1}$ of the set of points in $Z(f)$ of vertical tangency, self-intersection of zeros sets (roots with multiplicity), or a singularity of some other kind. Having constructed $\mathcal{G}$, the first-stage CAD

\footnotetext{
5 This notion is somewhat different than the notion of realizable sign conditions, where one can have several connected components representing the same sign condition.
} 
is obtained as the arrangement $\mathcal{A}(\{f\} \cup \mathcal{G})$ in $\mathbb{R}^{d}$, where the polynomials in $\mathcal{G}$ are now regarded as $d$-variate polynomials, that is, we lift them in the $x_{d}$-direction; the geometric interpretation of the lifting operation is to erect a "vertical wall" in $\mathbb{R}^{d}$ over each zero set within $\mathbb{R}^{d-1}$ of a $(d-1)$-variate polynomial from $\mathcal{G}$, and the first-stage CAD is the arrangement of these vertical walls plus $Z(f)$. It follows by construction that the cells of $\mathcal{A}(\{f\} \cup \mathcal{G})$ are vertical stacks of "cylindrical" cells. In more detail, for each cell $\tau$ of $\mathcal{A}(\{f\} \cup \mathcal{G})$, there is unique cell $\varphi$ of the $(d-1)$-dimensional arrangement $\mathcal{A}(\mathcal{G})$ in $\mathbb{R}^{d-1}$ such that one of the following two cases occur: (i) $\tau=\{(x, \xi(x)\} \mid x \in \varphi\}$, where $\xi: \varphi \rightarrow \mathbb{R}$ is a continuous semi-algebraic function (i.e., $\tau$ is the graph of $\xi$ over $\varphi$ ); or (ii) $\tau=\left\{(x, t) \mid x \in \varphi, t \in\left(\xi_{1}(x), \xi_{2}(x)\right)\right\}$, where $\xi_{i}, i=1,2$ is a continuous semi-algebraic function on $\varphi$, the constant function $\varphi \rightarrow\{-\infty\}$, or the constant function $\varphi \rightarrow\{+\infty\}$, and $\xi_{1}(x)<\xi_{2}(x)$ for all $x \in \varphi$ (i.e., $\tau$ is a cylindrical cell over $\varphi$ bounded from below (resp. above) by the graph of $\xi_{1}$ (resp. $\left.\xi_{2}\right)$ ). As stated in [2], the total number of cells in $\mathcal{A}(\{f\} \cup \mathcal{G})$ is $D^{O(d)}$, and each of them is a semi-algebraic set defined by $D^{O\left(d^{4}\right)}$ polynomials of degree $D^{O\left(d^{3}\right)}$ (this is, in fact, an application of Proposition 3).

For a cell $\varphi$ of the $(d-1)$-dimensional arrangement $\mathcal{A}(\mathcal{G})$, let $V(\varphi)$ be the stack of cells of $\mathcal{A}(\{f\} \cup \mathcal{G})$ over $\varphi$, i.e., the set of cells that project to $\varphi$.

We note that the sign condition of $\mathcal{P}$ is the same for all points in a cell of $\mathcal{A}(\{f\} \cup \mathcal{G})$, i.e., each cell lies in the realization of a single sign condition of $\mathcal{P}$. It thus suffices to find the cell of $\mathcal{A}(\{f\} \cup \mathcal{G})$ that contains the first intersection point of a vertical ray with a set of $\mathcal{S}$ in order to find the sign condition of $\mathcal{P}$ whose realization contains such a point. We construct $\mathrm{DS}_{1}, \mathrm{DS}_{2}$ on the cells of $\mathcal{A}(\{f\} \cup \mathcal{G})$ to quickly determine the desired cell.

The structure $\mathbf{D S}_{1}$. Fix a cell $\tau$ of $\mathcal{A}(\{f\} \cup \mathcal{G})$. DS 1 is used to determine whether a query ray $\rho_{q}$ whose source point lies in $\tau$ intersects any set of $\mathcal{S}$ inside $\tau$.

For each input set $S \in \mathcal{S}$ that intersects $\tau$, let $\uparrow S$ be the set of points $x$ in $\mathbb{R}^{d}$ such that the vertical ray $\rho_{x}$ intersects $S \cap \tau$, i.e., $\uparrow(S)$ is the union of the rays in the $\left(-x_{d}\right)$-direction emanating from the points of $S \cap \tau$. $\uparrow S$ is a semi-algebraic set whose complexity depends only on $b, d$, and $D$. Let $\uparrow \mathcal{S}_{\tau}=\{\uparrow S \mid S \in \mathcal{S}, S \cap \tau \neq \emptyset\}$. $\uparrow \mathcal{S}_{\tau}$ can be computed in $O(n)$ time. Using Theorem 12, we process $\uparrow \mathcal{S}$ into a data structure $\operatorname{DS}_{1}(\tau)$ of size $O\left(n^{d+\varepsilon}\right)$ so that for a query point $q \in \mathbb{R}^{d}$, we can determine in $O(\log n)$ time whether $q \in \bigcup \uparrow S$, i.e., whether $\rho_{q}$ intersects any set of $\mathcal{S}$ within $\tau$. We construct $\operatorname{DS}_{1}(\tau)$ for all cells $\tau$ of $\mathcal{A}(\{f\} \cup \mathcal{G})$. The total size of the data structure, summed over all cells of $\mathcal{A}(\{f\} \cup \mathcal{G})$, is $O\left(n^{d+\varepsilon}\right)$, and it can be constructed in $O\left(n^{d+\varepsilon}\right)$ randomized expected time.

The structure $\mathbf{D S}_{2}$. Fix a cell $\tau$ of $\mathcal{A}(\{f\} \cup \mathcal{G})$. $\mathrm{DS}_{2}$ is used to determine whether a line parallel to the $x_{d^{-}}$axis intersects any set of $\mathcal{S}$ inside $\tau$.

For each input set $S \in \mathcal{S}$ that intersects $\tau$, let $\downarrow S_{\tau}$ be the projection of $S \cap \tau$ onto the hyperplane $x_{d}=0$. For a point $q \in \mathbb{R}^{d}$, the vertical line (parallel to the $x_{d}$-axis) through $q$ intersects $S$ inside $\tau$ if and only if $\downarrow q \in \downarrow S_{\tau}$ (where $\downarrow q$ is the projection of $q$ onto the hyperplane $\left.x_{d}=0\right) . \downarrow S_{\tau}$ is a $(d-1)$-dimensional semi-algebraic set whose complexity depends only on $b$ and $D$. Let $\downarrow \mathcal{S}_{\tau}=\left\{\downarrow S_{\tau} \mid S \in \mathcal{S}, S \cap \tau \neq \emptyset\right\}$. $\downarrow \mathcal{S}_{\tau}$ can be constructed in $O(n)$ time. Using Theorem 12, we process $\downarrow \mathcal{S}_{\tau}$ into a data structure $\mathrm{DS}_{2}(\tau)$ of size $O\left(n^{d-1+\varepsilon}\right)$ so that, for a query point $q \in \mathbb{R}^{d}$, we can determine in $O(\log n)$ time whether $\downarrow q \in \bigcup \downarrow \mathcal{S}_{\tau}$, i.e., whether the vertical line through $q$ intersects any set of $\mathcal{S}$ inside $\tau$. We construct $\operatorname{DS}_{2}(\tau)$ for all cells of $\mathcal{A}(\{f\} \cup \mathcal{G})$. The total size of the data structure, summed over all cells of $\mathcal{A}(\{f\} \cup \mathcal{G})$, is $O\left(n^{d-1+\varepsilon}\right)$, and it can be constructed in $O\left(n^{d-1+\varepsilon}\right)$ randomized expected time.

Answering a query. Given a query point $q \in \mathbb{R}^{d}$, we determine the cell of $\mathcal{A}(\{f\} \cup \mathcal{G})$ that contains the first intersection point of $\rho_{q}$ with a set of $\mathcal{S}$ as follows. First, we determine the cell $\tau$ of $\mathcal{A}(\{f\} \cup \mathcal{G})$ that contains the query point $q$. Using $\mathrm{DS}_{1}(\tau)$, we determine in $O(\log n)$ 
time whether $\rho_{q}$ intersects $\mathcal{S}$ inside $\tau$. If the answer is yes, then $\tau$ is the desired cell. So assume that the answer is no. Let $\varphi$ be the cell of the $(d-1)$-dimensional arrangement $\mathcal{A}(\mathcal{G})$ such that $\downarrow q \in \varphi$. Let $V(\varphi)=\left\langle\tau_{1}, \ldots, \tau_{r}\right\rangle$ be the stack of cells over $\varphi$, and let $\tau=\tau_{i}$ for some $i \leq k$. We visit the cells of $V(\tau)$ one by one in order, starting from $\tau_{i+1}$ until we find a cell $\tau_{j}$ such that $\downarrow q \in \bigcup \downarrow \mathcal{S}_{\tau_{j}}$. Since $q$ lies below $\tau_{j}, \rho_{q}$ intersects $\mathcal{S}$ inside $\tau_{j}$ if and only if $\downarrow q \in \bigcup \downarrow\left(\mathcal{S}_{\tau_{j}}\right)$. If there is no such cell, we conclude that $\rho_{q}$ does not intersect $\mathcal{S}$. Otherwise $\tau_{j}$ is the cell of $\mathcal{A}(\{f\} \cup \mathcal{G})$ that contains the first intersection point of $\rho_{q}$ with a set of $\mathcal{S}$.

Putting everything together we obtain the following.

- Theorem 14. Let $\mathcal{S}$ be a collection of $n$ semi-algebraic sets in $\mathbb{R}^{d}$, each of complexity at most $b$ for some constant $b>1$. S can be preprocessed, in $O\left(n^{d+\varepsilon}\right)$ randomized expected time, into a data structure of size $O\left(n^{d+\varepsilon}\right)$, for any constant $\varepsilon>0$, so that a vertical ray-shooting query can be answered in $O\left(\log ^{2} n\right)$ time.

\subsection{Cutting algebraic curves into pseudo-segments}

Sharir and Zahl [15] presented a technique for cutting algebraic plane curves into pseudosegments, by lifting curves into three dimensions. More precisely, they prove that $n$ nonoverlapping algebraic curves of bounded degree $d$ can be cut into $O\left(n^{3 / 2} \log ^{O(1)} n\right)$ Jordan arcs so that each pair of arcs intersect in at most one point. Their procedure exploits Proposition 2 for algebraic curves in three dimensions; see [15, Theorem 1.1]. Theorem 11 can be used to prove a slightly weaker constructive and efficient variant of the above result, for a sketch of the proof see the full version of this paper [1].

Theorem 15. Let $\mathcal{C}$ be a set of $n$ algebraic plane curves, each of degree at most d, with no two sharing a common component. Then $\mathcal{C}$ can be cut into $O\left(n^{3 / 2+C_{d} / \log \log n}\right)$ Jordan arcs, where $C_{d}$ is a constant that depends on $d$, so that each pair of arcs intersect in at most one point. This cutting can be computed in randomized expected time $O\left(n^{3 / 2+C_{d} / \log \log n}\right)$.

By replacing Theorem 1.1 in [15] with our Theorem 15, we obtain effective and efficient version of all of the subsequent results in [15].

\section{- References}

1 Pankaj K. Agarwal, Boris Aronov, Esther Ezra, and Joshua Zahl. An Efficient Algorithm for Generalized Polynomial Partitioning and Its Applications. CoRR, abs/1812.10269, 2018. arXiv: 1812.10269.

2 Pankaj K. Agarwal, Jivr'i Matouvsek, and Micha Sharir. On Range Searching with Semialgebraic Sets. II. SIAM J. Comput., 42(6):2039-2062, 2013. doi:10.1137/120890855.

3 P.K. Agarwal. Simplex range searching and its variants: A review. A Journey Through Discrete Mathematics, pages 1-30, 2017.

4 Boris Aronov, Esther Ezra, and Joshua Zahl. Constructive Polynomial Partitioning for Algebraic Curves in $\mathrm{R}^{3}$ with Applications. In Proceedings of the Thirtieth Annual ACM-SIAM Symposium on Discrete Algorithms, SODA 2019, San Diego, California, USA, January 6-9, 2019, pages 2636-2648, 2019. doi:10.1137/1.9781611975482.163.

5 Sal Barone and Saugata Basu. Refined Bounds on the Number of Connected Components of Sign Conditions on a Variety. Discrete \& Computational Geometry, 47(3):577-597, 2012. doi:10.1007/s00454-011-9391-3.

6 S. Basu, R. Pollack, and M.F. Roy. Algorithms in Real Algebraic Geometry, volume 10. Springer Verlag, Berlin-Heidelberg, 2006.

7 Saugata Basu. Algorithms in Real Algebraic Geometry: A Survey. CoRR, abs/1409.1534, 2014. arXiv: 1409.1534. 
8 J. Bochnak, M. Coste, and M.F. Roy. Géométrie algébrique réelle (Second edition in english: Real Algebraic Geometry), volume 12(36). Springer Verlag, Berlin-Heidelberg, 1998.

9 Bernard Chazelle, Herbert Edelsbrunner, Leonidas J. Guibas, and Micha Sharir. A Singly Exponential Stratification Scheme for Real Semi-Algebraic Varieties and its Applications. Theor. Comput. Sci., 84(1):77-105, 1991. doi:10.1016/0304-3975(91)90261-Y.

10 J. Fox, J. Pach, A. Sheffer, A. Suk, and J. Zahl. A semi-algebraic version of Zarankiewicz's problem. J. Eur. Math. Soc., 19(6):1785-1810, 2017.

11 L. Guth. Polynomial partitioning for a set of varieties. Math. Proc. Camb. Philos. Soc., 159(3):459-469, 2015.

12 L Guth and N. Katz. On the Erdos distinct distance problem in the plane. Ann. of Math., 181:155-190, 2015.

13 S. Har-Peled. Geometric Approximation Algorithms, volume 173. AMS Press, Boston, MA, 2011.

14 Vladlen Koltun. Almost tight upper bounds for vertical decompositions in four dimensions. $J$. ACM, 51(5):699-730, 2004. doi:10.1145/1017460.1017461.

15 Micha Sharir and Joshua Zahl. Cutting algebraic curves into pseudo-segments and applications. J. Comb. Theory, Ser. A, 150:1-35, 2017. doi:10.1016/j.jcta.2017.02.006.

16 V.N. Vapnik and A.Y. Chervonenkis. On the uniform convergence of relative frequencies of events to their probabilities. Theory Probab. Appl., 16:264-280, 1971.

17 H.E. Warren. Lower bound for approximation by nonlinear manifolds. Trans. Amer. Math. Soc., 133:167-178, 1968. 